Pacific Journal of Mathematics

EXTENSIONS OF A FOURIER MULTIPLIER THEOREM OF 


\title{
EXTENSIONS OF A FOURIER MULTIPLIER THEOREM OF PALEY
}

\author{
JOHN FOURNIER
}

\begin{abstract}
Let $A$ be the class of continuous power series on the unit circle $T$, that is those continuous functions $f$ whose Fourier coefficients $\hat{f}(n)$ are 0 for negative indices $n$. It is known that the most that can be said about the size of the coefficients of such $f$ is that they are square summable. For instance Paley proved the following: Suppose that $\sum_{0}^{\infty}|w(n)|^{2}=\infty$. Then there is an $f$ in $A$ with $\sum_{0}^{\infty}|\hat{f}(n) w(n)|=\infty$. In other words the $l^{2}$ sequences are the only multipliers which map $A$ into the class of absolutely convergent power series.
\end{abstract}

The main result of this paper is that Paley's theorem can be generalized as follows: Let $G$ be a compact Abelian group with a partially ordered dual group $\Gamma$. Denote by $A$ the class of continuous functions $f$ on $G$ whose Fourier coefficients $\hat{f}(\gamma)$ vanish off the nonnegative cone $S$ of $\Gamma$. Let $E$ be a totally ordered subset of $S$ and $w$ be a function defined on $E$ which is not square summable. Then $\sum_{E}|\hat{f}(\gamma) w(\gamma)|=\infty$ for some $f$ in $A$.

The class $A$ when $\Gamma$ is in fact a totally ordered group is a frequently considered generalization of the algebra of continuous power series. In this situation $S$ itself is totally ordered so that $\sum_{S}|w(\gamma)|^{2}<\infty$, whenever $\sum|\hat{f}(\gamma) w(\gamma)|<\infty$ for all $f$ in $A$. This was obtained for $G=T^{n}$ by Helson [4] and in general by Rudin [8, p. 222]. Their proofs differed from Paley's although his method can be made to work in the situations they considered.

Now the power series discussed in the first paragraph are the restrictions to the circle of those functions which are continuous on the closure of the unit dise and analytic in its interior. From this point of view it would be natural, when $G=T^{2}$, to let $A$ be the class of restrictions, to the distinguished boundary of the unit bidisc, of functions which are continuous on the closure and analytic in the interior of the bidisc. These are precisely the continuous functions on $T^{2}$ whose Fourier coefficients $\hat{f}(m, n)$ vanish off the first quadrant $S$ of $Z^{2}$. The full analogue of Paley's theorem would be that every sequence $w$ with the Paley multiplier property, $\sum|w(N) \hat{f}(N)|<\infty$ for all $f$ in $A$, is square summable.

It is not known whether this strong version of theorem holds. The Helson-Rudin proofs for the case when $S$ is a half space depend on a property of the analytic projection $L$ taking trigonometric polynomials $\sum_{\Gamma} \hat{f}(\gamma) \gamma(x)$ into $\sum_{S} \hat{f}(\gamma) \gamma(x)$. Specifically, $\|L f\|_{p} \leqq K_{p}\|f\|_{1}$ 
for $p<1$. The corresponding projection when $S$ is the first quadrant does not have this property [12, Th. 4] and [13, p. 208].

Except for this, however, the above mentioned proofs work in the double power series case. A simple counterexample to the full analogue of Paley's theorem would provide a simple proof that the double analytic projection is not bounded from $L^{1}$ to $L^{p}$ for any $p<1$. As Helson observed, by a theorem of Bohr [2, p. 468, Th. 5], there are Paley multipliers on power series in infinitely many variables which do not even tend to 0 ; so the infinite dimensional version of Paley's theorem is false. This paper is the result of an attempt to settle the question for two or more variables.

What our main theorem says about Paley multipliers $w$ on double power series is that $\sum_{k=1}^{\infty}\left|w\left(N_{k}\right)\right|^{2}<\infty$ for any sequence $\left\{N_{k}\right\}_{k=1}^{\infty}$, of pairs of nonnegative integers, which is increasing in the strong sense that the $N_{k}$ are distinct and the sequences of first and second components are nondecreasing. It follows easily that all such Paley multipliers $w$ tend to 0 but perhaps not fast enough to make $\sum_{s}|w(N)|^{2}<$ $\infty$. So it is still not known if the only Paley multipliers on double power series are square summable. The proof of the main theorem does not involve properties of the analytic projection, however, and this suggests that Paley's theorem may not be as closely related to the boundedness of the projection as the previous proofs suggest.

As we shall see in $\S 3$, Paley multipliers can be thought of as coefficients in a semi-lacunary series on a somewhat larger group than $G$. The proof of the main theorem takes advantage of this fact and the method can be applied to lacunary Fourier series in other situations. In order to present the idea in a simple setting, we begin in $\S 2$ with such an application to semi-lacunary trigonometric series. In $\S 3$ we use the same general approach to prove the main theorem. Section 4 contains a discussion of Paley multipliers on power series in several variables; a number of special results not depending on the main theorem are obtained. In $\S 5$, we investigate Bohr sets, that is those subsets of $S$ whose characteristic functions are Paley multipliers. It turns out that all such sets are finite unions of sets in each of which no two elements are related under the partial ordering of $\Gamma$. Finally, in $\S 6$, we return to the subject of Fourier series whose restrictions to $S$ are lacunary and obtain some information about such series from our main theorem.

Notation and terminology have been taken from [8], which is a good source for the facts which we shall assume in what follows.

2. We begin with an illustration of our method in a simple setting.

THEOREM 1. Let $E=\left\{m_{n}\right\}_{n=1}^{\infty}$ be a set of positive integers with 
$m_{n+1}>2 m_{n}$ for all $n$. Suppose that $f$ is a function in $L^{1}(T)$ with $\hat{f}(m)=0$ for all nonnegative $m$ which do not belong to $E$. Then $\sum_{E}|\hat{f}(m)|^{2} \leqq 4\|f\|_{1}^{2}$.

Proof. We can assume that $\|f\|_{1}=1$. Factor $f=g \bar{h}$ where $g$ and $h$ are in $L^{2}(T)$ with $\|g\|_{2}=\|h\|_{2}=1$.

Then $\hat{f}(m)=1 / 2 \pi \int_{-\pi}^{\pi} g(\theta) \bar{h}(\theta) \exp (-i m \theta) d \theta$

$$
\text { i.e., } \quad \hat{f}(m)=\left\langle g, \chi^{m} n\right\rangle
$$

where $\chi(\theta)=\exp (i \theta)$ and $\langle.,$.$\rangle is the usual inner product in the Hil-$ bert space $L^{2}(T)$.

By assumption the inner product in (1) is 0 for most nonnegative $m$. The theorem is a consequence of the following result about such inner products.

Lemma 2. Let $H$ be a Hilbert space and $M_{1} \subset M_{2} \subset \cdots \subset M_{N}$ be closed subspaces of $H$. Let $A_{1}, A_{2}, \cdots, A_{N}$ be unitary linear operators on $H$ with $A_{1} M_{1} \subset A_{2} M_{2} \subset \cdots \subset A_{N} M_{N}$. Suppose that $g$ and $h$ are elements of $H$ satisfying:

(i) $A_{n} h \in A_{n+1} M_{n+1}$ for $n=1,2, \cdots, N-1$

(ii) $g$ is orthogonal to the subspaces $A_{n+1} M_{n}$ for $n=1,2, \cdots$, $N-1$.

Then $\sum_{1}^{N}\left|\left\langle g, A_{n} h\right\rangle\right|^{2} \leqq 4\|g\|^{2} \cdot\|h\|^{2}$.

To prove the theorem let $H$ be $L^{2}(T)$ and take $M_{n}$ to be the closed subspace of $L^{2}(T)$ generated by $\left\{\chi^{m} h \mid-m_{n} \leqq m<0\right\}$. Clearly

$$
M_{1} \subset M_{2} \subset \cdots \subset M_{n} \subset M_{n+1} \subset \cdots .
$$

Define $A_{n}$ by $A_{n} k=\chi^{m_{n}} k$ for all $k$ in $H$.

The subspaces $A_{n} M_{n}$ are the closed linear spans in $L^{2}(T)$ of $\left\{\chi^{m} h \mid 0 \leqq m<m_{n}\right\}$. So, $A_{1} M_{1} \subset A_{2} M_{2} \subset \cdots \subset A_{n} M_{n} \subset A_{n+1} M_{n+1} \subset \cdots$. Also as $m_{n}<m_{n+1}, A_{n} h \in A_{n+1} M_{n+1}$ for all $n$.

Finally $A_{n+1} M_{n}$ is the closed subspace generated by

$$
\left\{\chi^{m} h \mid m_{n+1}-m_{n} \leqq m<m_{n+1}\right\} .
$$

Now $m_{n+1}-m_{n}>2 m_{n}-m_{n}=m_{n}$ so that $m_{n+1}-m_{n} \leqq m<m_{n+1}$ implies $m_{n}\left\langle m<m_{n+1}\right.$. For such $m,\left\langle g, \chi^{m} h\right\rangle=\hat{f}(m)=0$ by assumption. Therefore $\langle g, k\rangle=0$ for every generator $k$ of $A_{n+1} M_{n}$ and hence for all $k$ in $A_{n+1} M_{n}$ and (ii) holds.

The lemma applies for any fixed $N$ to yield

$$
\sum_{n=1}^{N}\left|\hat{f}\left(m_{n}\right)\right|^{2}=\sum_{1}^{N}\left|\left\langle g, A_{n} h\right\rangle\right|^{2} \leqq 4 .
$$


Therefore $\sum_{n=1}^{\infty}\left|\hat{f}\left(m_{n}\right)\right|^{2} \leqq 4$.

Proof of Lemma 2. Again normalize by assuming $\|g\|=\|h\|=1$. By $M_{0}$ we shall mean the subspace of $H$ consisting of 0 alone.

For $n=0,1, \cdots, N$ let $k_{n}$ be the orthogonal projection of $h$ onto $M_{n}$. Then the sequence $k_{1}-k_{0}, k_{2}-k_{1}, \cdots, k_{N}-k_{N-1}$ is orthogonal and

$$
\sum_{1}^{N}\left\|k_{n}-k_{n-1}\right\|^{2}=\left\|k_{N}-k_{0}\right\|^{2} \leqq 1 .
$$

Now for each $n, A_{n}\left(h-k_{n}\right)$ is orthogonal to $A_{n} M_{n}$. But for $m<n, A_{m} h \in A_{m+1} M_{m+1} \subset A_{n} M_{n}$, and $A_{m} k_{m} \in A_{m} M_{m} \subset A_{n} M_{n}$. So for distinct $m$ and $n, A_{n}\left(h-k_{n}\right)$ and $A_{m}\left(h-k_{m}\right)$, are orthogonal with norm at most 1 .

Write $\left\langle g, A_{n} h\right\rangle=\left\langle g, A_{n}\left(h-k_{n}\right)\right\rangle+\left\langle g, A_{n}\left(k_{n}-k_{n-1}\right)\right\rangle+\left\langle g, A_{n} k_{n-1}\right\rangle=$ $a_{n}+b_{n}+c_{n}$ say.

By (ii) $c_{n}=0$ for all $n$.

By Bessel's inequality,

$$
\sum_{1}^{N}\left|a_{n}\right|^{2} \leqq\|g\|^{2}=1
$$

Finally $\left|b_{n}\right| \leqq\|g\| \cdot\left\|A_{n}\left(k_{n}-k_{n-1}\right)\right\|=\left\|k_{n}-k_{n-1}\right\|$ so that by $(2)$ $\sum_{1}^{N}\left|b_{n}\right|^{2} \leqq 1$.

The triangle inequality for $l^{2}$ yields $\left[\sum_{1}^{N}\left|\left\langle g, A_{n} h\right\rangle\right|^{2}\right]^{1 / 2} \leqq 2$.

Results like Theorem 1 are well known for lacunary series, i.e., series with $\hat{f}(m)=0$ for all $m$ off $E[14$, p. 205, Remark (a)]. The fact that the same is true for semi-lacunary series is implicit in an argument of Rudin, [9, §5.7], and seems to be well known among Fourier analysts. So the novelty of Theorem 1 lies in the method of proof rather than the conclusion. On the other hand, the most general situation in which our method works seems different from the one in which the usual technique works; we shall compare them in $\S 6$.

For the moment, let us remark that a simple modification of the above handles the case when, for some $\lambda$ strictly between 1 and 2 , $m_{n+1}>\lambda m_{n}$ for all $n$. It turns out that if $f$ is as in Theorem 1 then $\sum_{E}|\hat{f}(m)|^{2} \leqq(\sqrt{k}+1)^{2}\|f\|_{1}^{2}$, where $k$ is an integer chosen so that $\lambda^{k} \geqq \lambda /(\lambda-1)$.

3. In what follows, $G$ will be a compact Abelian group and $\Gamma$ will be the dual group of $G$, with the group operations written additively. $S$ will denote a semigroup in $\Gamma$ which contains 0 . We let $A$ be the algebra of continuous functions $f$ on $G$ for which $\hat{f}(\gamma)$ is 0 off $S$. For definiteness, the reader may find it convenient to imagine that $G=T^{2}, \Gamma=Z^{2}$, and that $S$ is the first quadrant in $Z^{2}$. 
Let $M$ be the class of Paley multipliers on $A$, that is those sequences $\{w(\gamma)\}_{\gamma \in S}$ with $\sum_{S}|\hat{f}(\gamma) w(\gamma)|$ finite for all $f$ in $A$. In other words for each $w$ in $M$, the mapping $f \rightarrow\{\hat{f}(\gamma) w(\gamma)\}_{\gamma \in S}$ sends $A$ into $l^{1}(S)$. In fact, by the closed graph theorem, this is a bounded linear operator. So $M$ is a normed linear space with the operator norm:

$$
\|w\|_{M}=\sup \sum_{S}|\hat{f}(\gamma) w(\gamma)| \quad\left(f \text { in } A \text { and }\|f\|_{\infty}=1\right) .
$$

Observe that if $v$ is a sequence with $|v(\gamma)| \leqq|w(\gamma)|$ for all $\gamma$ in $S$ then $\|v\|_{M} \leqq\|w\|_{M}$. In particular this is true if $v$ is a truncation of $w$ which agrees with $w$ on part of $S$ and is 0 elsewhere.

For any sequence $\varepsilon(\gamma)= \pm 1$ we have that $\left|\sum_{s} \varepsilon(\gamma) f(\gamma) w(\gamma)\right| \leqq$ $\|w\|_{M} \cdot\|f\|_{\infty}$. Therefore the mapping $f \rightarrow \sum_{S} \varepsilon(\gamma) w(\gamma) \hat{f}(\gamma)$ is a bounded linear functional on $A$ of norm no greater than $\|w\|_{M}$. By the Hahn-Banach theorem it has a norm preserving extension to all of the continuous functions on $G$. This means that there is a bounded regular Borel measure $\mu$ on $G$ with $\|\mu\| \leqq\|w\|_{M}$ and

$$
\sum_{S} \varepsilon(\gamma) w(\gamma) \hat{f}(\gamma)=\int_{G} f(x) d \mu(-x)
$$

for all $f$ in $A$. Taking $f=\gamma$ for any $\gamma$ in $S$ we obtain:

$$
\varepsilon(\gamma) w(\gamma)=\int_{G} \gamma(x) d \mu(-x)=\int_{G} \gamma(-x) d \mu(x)=\hat{\mu}(\gamma) .
$$

The property that for every choice of signs $\varepsilon(\gamma)$ there are measures $\mu$ satisfying (1) characterizes $M$ and was used by Helson and Rudin in their proofs of Paley's theorem ([4] and [8, p. 222]).

Now $S$ induces a partial ordering of $\Gamma$ under the rule: $\gamma_{1} \leqq \gamma_{2}$ if and only if $\gamma_{2}-\gamma_{1} \in S$. The order relation is transitive and invariant under addition but it may happen that $\gamma_{1} \leqq \gamma_{2} \leqq \gamma_{1}$ without $\gamma_{1}=\gamma_{2}$.

We can now state and prove our main theorem.

THEOREM 3. Let $w \in M$ and $E \subset S$ be totally ordered under the order induced by $S$. Then

$$
\sum_{E}|w(\gamma)|^{2} \leqq 4\|w\|_{M}^{2}
$$

Proof. It is enough to prove the theorem for $\|w\|_{M}=1$ and $E$ finite. Let $\gamma_{1} \leqq \gamma_{2} \leqq \cdots \leqq \gamma_{N}$ be the elements of $E$. Denote by $v$ the truncation of $w$ to $E: v(\gamma)=w(\gamma)$ if $\gamma \in E$ and $v(\gamma)=0$ otherwise. As observed above $\|v\|_{M} \leqq 1$.

Let $\varepsilon(\gamma)$ be any sequence of \pm 1 on $S$. There is a bounded regular Borel measure $\mu$ on $G$ with $\|\mu\| \leqq 1$ and $\hat{\mu}(\gamma)=\varepsilon(\gamma) v(\gamma)$ for all 
$\gamma$ in $S$.

Fix $K>1$. As $E$ is finite there is a trigonometric polynomial $P$ on $G$ with $\|P\|_{1} \leqq K^{2}$ and $\hat{P}(\gamma)=1$ on $E$ [8, Th. 2.6.8]. Then $f \equiv$ $P * \mu$ is a trigonometric polynomial with the following properties:

(2) $\|f\|_{1} \leqq K^{2}, \hat{f}(\gamma)=\varepsilon(\gamma) w(\gamma)$ on $E$ and 0 elsewhere in $S$, and $\hat{f}(\gamma)=0$ off the support of $\hat{P}$.

It is a theorem of Littlewood [5] that, if for each choice of signs there is an $f$ satisfying (2) and with $\hat{f}=0$ off $E$, then $\sum_{E}|w(\gamma)|^{2} \leqq$ $B K^{4}$ where $B$ is a fixed constant. Our problem is to reach the same conclusion assuming only that $\hat{f}(\gamma)=0$ on the rest of $S$.

In order to make use of the random signs $\varepsilon(\gamma)$ in the above, we introduce the Rademacher functions. Let $Q$ be the Cartesian product of $N$ copies of $Z_{2}$, the additive cyclic group of order 2. Denote the elements of $Q$ by $t=\left(t_{1}, \cdots, t_{n}\right)$ with each $t_{j}=0$ or 1 . Define the $n$ 'th Rademacher function $r_{n}$ by

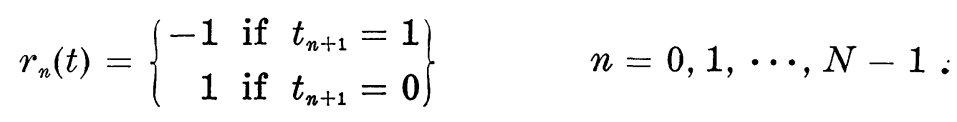

By (2), for each $t$ in $Q$ we can find a trigonometric polynomial $f(t, x)$ on $G$ so that $\int_{G}|f(t, x)| d x \leqq K^{2},[f(t, \cdot)]^{\wedge}(\gamma)=0$ off the support of $\hat{P}$ and

$$
[f(t, \cdot)]^{\wedge}(\gamma)=\left\{\begin{array}{l}
r_{n-1}(t) w\left(\gamma_{n}\right) \text { if } \gamma=\gamma_{n} \in E \\
0 \text { for all other } \gamma \text { in } S
\end{array}\right\} .
$$

Letting $d t$ be the Haar measure on $Q$ which assigns mass $2^{-N}$ to each point, we get $\int_{Q} \int_{G}|f(t, x)| d x d t \leqq K^{2}$. That is, if we assume for the moment that $f$ is a measurable function on $Q \times G$, then $f \in L^{1}(Q \times G)$ with norm no greater than $K^{2}$.

In fact, $f$ is a trigonometric polynomial on $Q \times G$, that is, a finite linear combination of continuous characters on $Q \times G$, but to be sure of this we must look at the set of such characters, i.e., the dual group of $Q \times G$.

To begin with, the complete set of characters on $Q$ is the set of Walsh functions $\psi(m)(t), m=0,1, \cdots, 2^{N}-1$ [3, pp. 376-377], which are defined as follows. We write

$$
m=2^{n_{1}}+\cdots+2^{n_{k}}, 0 \leqq n_{1}<n_{2}<\cdots<n_{k}
$$

and let $\psi(m)(t)=r_{n_{1}}(t) \cdot r_{n_{2}}(t) \cdots r_{n_{k}}(t)$, with the convention that $\psi(0)(t) \equiv 1$. $R$, the dual group of $Q$ is the set of all such functions, under multiplication.

The dual group of $Q \times G$ is $R \times \Gamma$ [8, Th. 2.2.2] so that the products $\psi(m)(t) \gamma(x)$ form the complete set of continuous characters 
on $Q \times G$.

For fixed $t, f(t, x)$ is a trigonometric polynomial on $G$ whose coefficients are 0 off the support of $\hat{P}$. As $Q$ and $R$ are finite, all functions on $Q$ are trigonometric polynomials and in particular $[f(t, \cdot)]^{\wedge}(\gamma)$ is a trigonometric polynomial on $Q$ for each $\gamma$. So, the finite $\operatorname{sum} f(t, x)=$ $\sum_{\gamma \in \operatorname{supp} \hat{P}} \gamma(x)[f(t, \cdot)]^{\wedge}(\gamma)$ is a trigonometric polynomial on $Q \times G$.

Write $f(t, x)=\sum_{m=0}^{2^{N}-1} \sum_{\gamma \in \Gamma} a(m, \gamma) \psi(m)(t) \gamma(x)$. Clearly $a(m, \gamma)=0$ unless $\gamma \in$ supp $\hat{P}$ and in view of (3) we have

$$
a(m, \gamma)=\left\{\begin{array}{l}
w\left(\gamma_{n}\right) \text { if } \gamma=\gamma_{n} \text { and } m=2^{n-1} \\
0 \text { otherwise for } \gamma \text { in } S
\end{array}\right\} .
$$

We have no information about $a(m, \gamma)$ when $\gamma$ is in supp $\hat{P}$ but not in $S$ but this will not matter.

The proof now proceeds much as in Theorem 1 with the products $\psi\left(2^{n-1}\right) \gamma_{n}$ playing the role of the thin set of characters $\left\{\chi^{m}\right\}_{n=1}^{\infty}$.

Factor $f(t, x)=g(t, x) \bar{h}(t, x)$ where $g, h \in L^{2}(Q \times G)$ and $\|g\|_{2}=$ $\|h\|_{2}=\|f\|_{1}^{1 / 2} \leqq K$.

For all $m, \gamma$

$$
\begin{aligned}
& a(m, \gamma)=\int_{Q} \int_{G} g(t, x) \bar{h}(t, x) \bar{\gamma}(x) \bar{\psi}(m)(t) d x d t, \\
& \text { i.e., } a(m, \gamma)=\langle g, \psi(m) \gamma h\rangle .
\end{aligned}
$$

We wish to apply Lemma 2 with $A_{n} k \equiv \psi\left(2^{n-1}\right) \gamma_{n} k$ for all $k$ in $L^{2}(Q \times G)$. Assume for a moment that subspaces $M_{n}$ can be chosen so that the hypotheses of the lemma hold. Then, in view of (4) and (5)

$$
\sum_{1}^{N}\left|w\left(\gamma_{n}\right)\right|^{2}=\sum_{1}^{N}\left|a\left(2^{n-1}, \gamma_{n}\right)\right|^{2}=\sum_{1}^{N}\left|\left\langle g, A_{n} h\right\rangle\right|^{2} \leqq 4 K^{2}
$$

which is what we want, as $K$ is any constant larger than 1 .

It remains to chose the $M_{n}$ so that the assumptions of the lemma are satisfied. This is the only part of the proof where the total ordering of $E$ is used.

Let $M_{n}$ be the closed linear span in $L^{2}(Q \times G)$ of $\{\psi(m) \gamma h \mid(m, \gamma) \neq$ $\left.(0,0), 0 \leqq m<2^{n},-\gamma_{n} \leqq \gamma \leqq 0\right\} . \quad$ As $\gamma_{1}<\gamma_{2}<\cdots<\gamma_{N}$ it is clear that $M_{1} \subset M_{2} \subset \cdots \subset M_{n}$.

The set $\left\{\psi(m) \mid 0 \leqq m<2^{n}\right\}$ is the subgroup of the Walsh functions generated by $r_{0}, r_{1}, \cdots, r_{n-1}$. Therefore, $A_{n} M_{n}$ is the closed subspace generated by $\left\{\psi(m) \gamma h \mid(m, \gamma) \neq\left(2^{n-1}, \gamma_{n}\right), 0 \leqq m<2^{n}, 0 \leqq \gamma \leqq \gamma_{n}\right\}$. Certainly $A_{1} M_{1} \subset A_{2} M_{2} \subset \cdots \subset A_{N} M_{N}$. Moreover

$$
A_{n} h=\psi\left(2^{n-1}\right) \gamma_{n} h \in A_{n+1} M_{n+1}
$$

for $n<N$ and (i) holds. Again this depends on the fact that $\gamma_{n} \leqq \gamma_{n+1}$. 
Finally if $m<2^{n}, r_{n}$ is not one of the factors of $\psi(m)$. So

$$
\psi(m) \psi\left(2^{n}\right)=\psi(m) r_{n}=\psi\left(m+2^{n}\right) .
$$

Therefore, $A_{n+1} M_{n}$ is the closed linear span of

$$
\left\{\psi(m) \gamma h \mid(m, \gamma) \neq\left(2^{n}, \gamma_{n+1}\right), 2^{n} \leqq m<2^{n+1}, \gamma_{n+1}-\gamma_{n} \leqq \gamma \leqq \gamma_{n+1}\right\} .
$$

Now when $m$ and $\gamma$ satisfy these restrictions, then $\gamma \in S$ as $\gamma_{n+1}-\gamma_{n} \geqq 0$, and by (5) and (4), $\langle g, \psi(m) \gamma h\rangle=a(m, \gamma)=0$. That is $\langle g, k\rangle=0$ for every generator $k$ of $A_{n+1} M_{n}$ and hence for every $k$ in $A_{n+1} M_{n}$. (ii) holds and the proof of the theorem is complete.

In fact the argument actually works under somewhat weaker assumptions than those above.

Definition. A set $I \subset \Gamma$ is called convex if $\left\{\gamma \mid \gamma_{1} \leqq \gamma \leqq \gamma_{2}\right\} \subset I$ whenever $\gamma_{1}$ and $\gamma_{2}$ are in $I$.

THEOREM 4. Let $I \subset \Gamma$ be convex and $w$ be a sequence defined on I. Define

$$
\|w\|_{I}=\sup \sum_{I}|\hat{f}(\gamma) w(\gamma)| \quad\left(f \in C_{I}(G),\|f\|_{\infty}=1\right),
$$

where $C_{I}(G)=\{f \in C(G) \mid \hat{f}(\gamma)=0$ off $I\}$. Let $E \subset I$ be totally ordered. Then $\sum_{E}|w(\gamma)|^{2} \leqq 4\|w\|_{I}^{2}$.

Outline of proof. The method of Theorem 3 works in this situation. The main change is that statements which held for all $\gamma$ in $S$ in the proof of Theorem 3 now hold for all $\gamma$ in $I$. Lemma 2 applies with $M_{n}$ taken to be the closed linear span in $L^{2}(Q \times G)$ of $\left\{\psi(m) \gamma h \mid(m, \gamma) \neq(0,0), 0 \leqq m<2^{n}\right.$, and $\left.\gamma_{1}-\gamma_{n} \leqq \gamma \leqq 0\right\}$. We omit the details.

In the special case $G=T, S=\{n \mid n \geqq 0\}, I=\left\{n \mid n_{1} \leqq n \leqq n_{2}\right\}, I$ itself is totally ordered and we conclude that $\sum_{I}|w(n)|^{2} \leqq 4\|w\|_{I}^{2}$. With the constant 4 replaced by a much larger one, this was obtained by Steckin [11, Lemma 2] as a consequence of Paley's theorem.

The definition of $\|w\|_{I}$ makes sense for any set $I$ and does not depend at all on $S$. Furthermore a set may be convex with respect to several orderings of $\Gamma$. For instance, let $G=T^{2}, \Gamma=Z^{2}, I=$ first quadrant in $Z^{2}$. We can take $S$ to be any quadrant and in each case $I$ is convex with respect to the order induced by $S$. So, $S$ plays an indirect role in Theorem 4 which may be restated as follows:

TheOREM 4'. Let $I$ be a subset of $\Gamma$. Define $\|w\|_{I}$ as before. Then $\sum_{E}|w(\gamma)|^{2} \leqq 4\|w\|_{I}^{2}$ for any set $E \subset I$ which is totally ordered 
under some ordering of $\Gamma$ with respect to which $I$ is convex.

4. We now treat the case of power series in a finite number of variables. That is, $G=T^{n}, \Gamma=Z^{n}$, and $S=\left\{N=\left(N_{1}, N_{2}, \cdots, N_{n}\right) \mid N_{j} \geqq\right.$ 0 for all $j$.

The main theorem tells us that, when $w$ is a Paley multiplier, 4 $\|w\|_{M}^{2}$ is a uniform bound on $\sum_{E}|w(N)|^{2}$ for all totally ordered subsets $E$ of $S$. Unfortunately, such sets $E$ are essentially one dimensional. For instance, the $N_{1}$ axis $\left\{N\right.$ in $S \mid N_{j}=0$ for $\left.j>1\right\}$ is a maximal totally ordered subset of $S$.

It is possible, however, to give bounds on $\sum_{D}|w(N)|^{2}$ for some sets $D$ which are not as thin as totally ordered sets. For simplicity we prove the following theorem only for the case $n=2$.

THEOREM 5. Fix an integer $L>0$ and let $D$ be the set

$$
\left\{N \mid L \leqq N_{1} \leqq 3 L \quad \text { and } \quad N_{2} \geqq 0\right\} \text {. }
$$

Then for every $w$ in $M, \Sigma_{D}|w(N)|^{2} \leqq 36\|w\|_{M}^{2}$.

Proof. Since truncation does not increase norms in $M$, assume that $w=0$ off $D$.

Let $K_{n}(\theta)$ be the Fejer kernel $\sum_{m=-n}^{n}[1-|m| /(n+1)] \exp (i m \theta)$. Put $K(\theta)=\exp (i 2 L \theta)\left[2 K_{2 L-1}(\theta)-K_{L-1}(\theta)\right]$. Then $(1 / 2 \pi) \int_{-\pi}^{\pi}|K(\theta)| d \theta \leqq$ $3, \hat{K}(m)=1$ if $L \leqq m \leqq 3 L$, and $\hat{K}(m)=0$ if $m<0$. Define a measure $\nu$ on $T^{2}$ by $\int_{T^{2}} f(\theta, \varphi) d \nu(-\theta,-\varphi)=(1 / 2 \pi) \int_{-\pi}^{\pi} f(\theta, 0) K(-\theta) d \theta$. Then $\|\nu\| \leqq 3, \hat{\nu}=1$ on $D$, and $\hat{\nu}(N)=0$ if $N_{1}<0$.

Now let $S_{1}$ be the half space $\left\{N \mid N_{2}>0\right.$, or $N_{2}=0$ and $\left.N_{1} \geqq 0\right\}$. $w$ can be thought of as a Paley multiplier on $C_{S_{1}}\left(T^{2}\right)$, the continuous functions whose coefficients vanish off $S_{1}$. For, suppose that $f$ is such a function. Let $g=f * \nu$. Then $g \in A$ and $\|g\|_{\infty} \leqq 3\|f\|_{\infty}$. Extend $w$ to $S_{1}$ by setting it equal to 0 on the rest of $S_{1}$. Now,

$$
\sum_{S_{1}}|\hat{f}(N) w(N)|=\sum_{D}|\hat{g}(N) w(N)| \leqq\|w\|_{M}\|g\|_{\infty} \leqq 3\|w\|_{M}\|f\| .
$$

So, as a multiplier on $C_{S_{1}}$, w has norm at most $3\|w\|_{M}$. It follows from the main theorem that

$$
\sum_{D}|w(N)|^{2}=\sum_{S_{1}}|w(N)|^{2} \leqq 36\|w\|_{M}^{2} \cdot
$$

The same kind of conclusion can be obtained in dimension $n$ for sets $D$ of the form:

$$
\left\{N \mid N_{n} \geqq 0 \text { and } L_{j} \leqq N_{j} \leqq 3 L_{j} \text { for } j=1,2, \cdots, n-1\right\}
$$


where $L_{1}, L_{2}, \cdots, L_{n-1}$ are any fixed nonnegative integers. Of course the last coordinate need not be the one that is free in the above.

As we shall see in the next section, the main theorem implies that, in the context of power series in a finite number of variables, $w(N) \rightarrow 0$ as $N \rightarrow \infty$ whenever $w \in M$. This fact can also be derived from the following lemma of Helson.

Lemma (Translation Lemma). Let $G$ be a compact Abelian group. Suppose that $\mu$ is a finite regular Borel measure on $G$ and $\left\{\gamma_{n}\right\}_{1}^{\infty}$ is a sequence of distinct elements of $\Gamma$. Define measures $\lambda_{n}$ by $d \lambda_{n}(x)=$ $\gamma_{n}(x) d \mu(x)$. Let $\lambda_{n} \rightarrow \sigma$ in the weak star topology. Then $\sigma$ is singular with respect to the Haar measure of $G$ [8, Lemma 3.5.1].

Theorem 6. With $G=T^{n}$ and $S$ as above, $w(N) \rightarrow 0$ as $N \rightarrow \infty$ for every Paley multiplier $w$.

Proof. Suppose that the theorem is false. Let $w$ have the property that $|w(N)| \geqq 1$ on an infinite set $B$ of $N^{\prime}$ s.

First assume that $B$ contains an infinite sequence $\left\{N^{(k)}\right\}_{1}^{\infty}$ such that for each $k$ and all $j, N_{j}^{(k)} \geqq k$. Then in fact the sequence can be chosen to be lacunary in the sense that for each $k$ and $j, N_{j}^{(k+1)}>$ $2 N_{j}^{(k)}$. Let $v$ be equal to 1 on this sequence and 0 elsewhere. As $v$ is dominated by $w$, it is a Paley multiplier.

Therefore there is finite regular Borel measure $\mu$ on $T^{n}$ so that $\hat{\mu}\left(N^{(k)}\right)=1$ for all $k$ and $\hat{\mu}(N)=0$ for all other $N$ in $S$. Consider the measures $\lambda_{k}$ defined by $d \lambda_{k}(x)=\exp \left(-i N^{(k)} \cdot x\right) d \mu(x)$. For all $k,\left\|\lambda_{k}\right\|=$ $\|\mu\|$, so that a subsequence of the $\lambda_{k}^{\prime} \mathrm{s}$ converges in the weak star sense to a measure $\sigma$.

$\hat{\lambda}_{k}(0)=1$ for all $k$, so that $\hat{\sigma}(0)=1$. For any $N \neq 0, \hat{\lambda}_{k}(N)=0$ for all large $k$. Hence $\hat{\sigma}(N)=0$ for $N \neq 0$. This means that $d \sigma$ is $d x$, the Haar measure on $T^{n}$. But by the translation lemma, $d \sigma$ is singular with respect to $d x$, a contradiction.

The preceding three paragraphs prove the theorem for the case $n=1$ as then any infinite $B$ would contain such a sequence $\left\{N^{(k)}\right\}$.

For $n>1$, we conclude that $B$ contains no such sequence. It follows that there is an integer $k$ for which the cone $\left\{N \mid N_{j}>k\right.$ for all $j$ \} does not intersect $B$. In other words $B$ is contained in the union of the $(k+1) \cdot n$ hyperplanes $\left\{N\right.$ in $\left.S \mid N_{j}=h\right\}$ where $j$ runs from 1 to $n$ and $h$ from 0 to $k$. The intersection of $B$ with one of these hyperplanes, for particular choices of $j$ and $h$, is infinite. Let $S_{1}$ be the positive cone in $Z^{n-1}$. Define a sequence $v$ on $S_{1}$ by

$$
v\left(N_{1}, N_{2}, \cdots, N_{n-1}\right)=w\left(N_{1}, N_{2}, \cdots, N_{j-1}, h, N_{j+1}, \cdots, N_{n-1}\right) .
$$

It is not hard to see the that $v$ is a Paley multiplier on $C_{S_{1}}\left(T^{n-1}\right)$ 
with $|v(N)| \geqq 1$ for infinitely many $N$ in $S_{1}$. The theorem follows by induction on the dimension $n$.

The idea for the application of the translation lemma in the above came from Rider's treatment of the infinitite dimensional case [7, §3]. In fact Rudin made a similar application in [9, Th. 4] to obtain the result in the one variable case.

So, for the case of power series in two or more variables Theorems 3,5 , and 6 provide a variety of restrictions which must be satisfied by any Paley multiplier. We now give an example for the case of two variables of a sequence which satisfies these restrictions but is not square summable. It resembles one given by Bohr in infinitely many variables [2, p. 468, Th. 5] and arose from a suggestion of Professor Walter Rudin.

For $m \geqq 0, n \geqq 0$, let $w(m, n)=1 /(m+n+1)$.

Observe that any totally ordered set $E$ intersects the line $l_{k}=$ $\{(m, n) \mid m+n=k\}$ in at most one point. For any such $E$,

$$
\sum_{E}|w(N)|^{2}=\sum_{k=0}^{\infty} \sum_{E \cap l_{k}}|w(N)|^{2} \leqq \sum_{0}^{\infty} 1 /(k+1)^{2}=\pi^{2} / 6 .
$$

Hence $w$ satisfies the conclusion of Theorem 3 .

Next let $D$ be any set of the type considered in Theorem 5. For $k<L, l_{k} \cap D$ is empty and for any $k, l_{k} \cap D$ has at most $2 L+1$ elements. Therefore,

$$
\begin{aligned}
\sum_{D}|w(N)|^{2} & =\sum_{k=L}^{\infty} \sum_{l_{k} \cap D}|w(N)|^{2} \leqq \sum_{L}^{\infty}(2 L+1)(k+1)^{-2} \\
& \leqq \frac{2 L+1}{L} \leqq 3
\end{aligned}
$$

and the conclusion of Theorem 5 holds for $w$.

Finally, it is clear that $w(N) \rightarrow 0$ as $N \rightarrow \infty$.

On the other hand,

$$
\sum_{S}|w(N)|^{2}=\sum_{k=0}^{\infty} \sum_{l_{k} \cap S}|w(N)|^{2}=\sum_{k=0}^{\infty}(k+1)(k+1)^{-2}=\infty .
$$

It is not known whether $w$ is a Paley multiplier sequence. This example shows, however, that in the context of power series in two as more variables, our results do not imply that $M=l^{2}(S)$. The question is therefore still open for the case of $n$ variables, $1<n<\infty$.

5. We modify a definition of Rider [7, p. 558].

Definition. Let $G, S$, and $A$ be as in $\S 3$. A subset $B$ of $S$ will 
be called a Bohr set if there is a constant $K$ so that $\sum_{B}|\hat{f}(\gamma)| \leqq$ $K\|f\|_{\infty}$ for all $f$ in $A$.

In other words, $B$ is a Bohr set if the sequence which is 1 on $B$ and 0 elsewhere in $S$ is a Paley multiplier.

The reason for the name Bohr set is the following theorem of Bohr [2, p. 468, Th. 5]. Let $G$ be the complete direct sum $T^{\omega}$ of countably many circles and $\Gamma$ be the direct sum $Z^{\infty}$, [8, § 8.7.9]. Let $S=\left\{N \in Z^{\infty} \mid N_{j} \geqq 0\right.$ for all $\left.j\right\}$ and let $A$ be the space of continuous functions on $T^{\omega}$ with coefficients supported by $S$. Let $B=\left\{N \in Z^{\infty} \mid N_{j}=\right.$ $\delta_{i j}$ for some $\left.i\right\}$. Then $\sum_{B}|\hat{f}(N)| \leqq\|f\|_{\infty}$ for all $f$ in $A$. Other examples of Bohr sets and an account of the connection with Dirichlet series appear in [7].

We use Theorem 3 to obtain necessary arithmetic conditions on Bohr sets.

THEOREM 7. Let $B$ be a Bohr set and $K$ the constant of the definition. Then every totally ordered subset of $B$ has at most $4 K^{2}$ elements.

Proof. By assumption the multiplier $w$ which is 1 on $B$ and 0 elsewhere has norm at most $K$. If $E \subset B$ is totally ordered, $\sum_{E} 1=$ $\sum_{E}|w(\gamma)|^{2} \leqq 4 K^{2}$.

Observe that the theorem certainly holds for Bohr's example $B$. Totally ordered subsets of $B$ have one element as no two elements of $B$ are related under the order induced by $S$.

Definition. A subset $B$ or $\Gamma$ will be called unrelated if no two elements of $B$ are related under the order induced by $S$.

Lemma 8. $A$ subset $B$ of $\Gamma$ contains no totally ordered set with more than $K$ elements if and only if $B$ is the union of at most $K$ unrelated sets.

Proof. It is obvious that such a union contains no totally ordered set with more than $K$ elements.

Conversely, suppose that the totally ordered subsets of $B$ have at most $K$ elements. Let $E$ be a totally ordered subset of $B$, maximal with respect to containment. We shall find a set $B_{1}$ consisting of exactly one minimal element from each such $E$. As $E$ is finite the set $F$ of minimal elements of $E$ is nonempty. By the maximality of $E$, $F$ is a maximal equivalence class in $B$ : i.e., for any $\gamma$ in $F, F=\left\{\gamma^{\prime}\right.$ in $\left.B \mid \gamma \leqq \gamma^{\prime} \leqq \gamma\right\}$. Thus if $E^{\prime}$ is another maximal totally ordered subset 
of $B$ and $F^{\prime}$ is the set of minimal elements of $E^{\prime}$ then either $F=F^{\prime}$ or $F$ and $F^{\prime}$ are disjoint. The axiom of choice yields a set $B_{1}$ consisting of one element from each such $F$, that is one minimal element from each such $E$. By the maximality of the $E$ 's, $B_{1}$ is unrelated. Moreover as every totally ordered subset of $B$ is contained in such an $E, B \sim B_{1}$ contains no totally ordered set with more than $K-1$ elements. The lemma follows by induction on $K$.

THEOREM 9. Every Bohr set is the union of at most $4 K^{2}$ unrelated sets, where $K$ is the constant in the definition of Bohr set.

Proof. Combine 7 and 8.

It can be shown that every unrelated subset of the positive cone $S$ of $Z^{n}$ is finite. This means that, for the case of power series in $n$ variables, Bohr sets are finite. This statement is equivalent to Theorem 6, as it is easy to see in any case that there is an infinite Bohr set in $S$ if and only if there is a Paley multiplier which does not tend to 0 . In fact we can use Theorem 7 in place of the Translation Lemma in the proof of Theorem 6. Simply observe that the lacunary sequence discussed in the second paragraph of the proof of Theorem 6 is increasing with respect to the order induced by $S$ and can have at most $4 K^{2}$ elements, contrary to the assumption that it is infinite. Therefore there is no such sequence and the last paragraph of the proof of Theorem 6 applies.

We now turn to the case of power series in infinitely many variables; i.e., $G$ is the complete direct sum $T^{\omega}, \Gamma$ is the direct sum $Z^{\infty}$ and $S=\left\{N \mid N_{j} \geqq 0\right.$ for all $\left.j\right\}$. Bohr's example shows that there are infinite Bohr sets in this case.

In $[7$, p. 560] Rider gives sufficient arithmetic conditions for a set to be a Bohr set: Let $B \subset S$ satisfy:

(c) the elements of $B$ are linearly independent over the integers.

(d) whenever $N \in S$ and $N=\sum_{1}^{k} \beta_{i} N^{(i)}$ where the $\beta_{i}$ are integers, $\sum_{1}^{k} \beta_{i}=1$, and the $N^{(i)} \in B$ for all $i$, then $N \in B$.

Then $B$ is a Bohr set.

It is easy to see that these conditions force any such $B$ to be unrelated. For if $N^{(1)}<N^{(2)}$ are in $B$, then by (d)

$$
M^{(k)} \equiv N^{(1)}+k\left(N^{(2)}-N^{(1)}\right)
$$

is in $B$ for all $k \geqq 0$. But $M^{(2)}+N^{(1)}-2 N^{(2)}=0$ contrary to (c).

On the other hand, an unrelated set need not be a Bohr set. For instance let $B_{k}=\left\{N\right.$ in $S \mid N_{j}=0$ unless $j=2 k-1$ or $2 k$, and $\left.\sum_{1}^{\infty} N_{j}=k\right\}$. Let $B=\bigcup_{1}^{\infty} B_{k}$. It is easy to see that $B$ is unrelated. Let $w$ be the sequence which is 1 on $B$ and 0 elsewhere. Apply 
Theorem $4^{\prime}$ with $I=S$, using the order induced by $\left\{N\right.$ in $Z^{\infty} \mid N_{j} \geqq 0$ for $j \neq 2 k$ and $\left.N_{2 k} \leqq 0\right\}$, to obtain: $k+1=\sum_{B_{k}}|w(N)|^{2} \leqq 4\|w\|_{M}^{2}$. Therefore $w$ is not a Paley multiplier and $B$ is not a Bohr set. We can modify this example so that it becomes an unrelated Sidon set which is not a Bohr set.

Nevertheless any set consisting of exactly one element from each $B_{k}$ satisfies (c) and (d) and is therefore a Bohr set. It is not clear whether every infinite unrelated set must contain an infinite Bohr set.

It is shown in [2] that there is a connection between Dirichlet series and power series in infinitely many variables. Theorem 9 can be restated as follows:

THEOREM. Suppose that $B$ is a set of positive integers so that there is a constant $K$, with $\sum_{B}|c(n)| \leqq K$ whenever there is a Dirichlet series $f(s+i t)=\sum_{1}^{\infty} c(n) n^{-s-i t}$ with $|f(s+i t)| \leqq 1$ for all $s>0$. Then $B$ is the union of at most $4 K^{2}$ sets in each of which no element divides any other.

6. Conclusions similar to Theorem 1 can be obtained under weaker assumptions. Once again $G, \Gamma, S$, and $A$ are as in $\S 3$.

Definition. A set $B \subset \Gamma$ is called a Sidon set if there is a constant $K$ so that $\sum_{B}|\hat{f}(\gamma)| \leqq K\|f\|_{\infty}$ for every trigonometric polynomial $f$ for which $\hat{f}$ is 0 off $B,[8, \S 5.7]$.

THEOREM 10. Let $B$ be a Sidon set and $I$ be a convex subset of $\Gamma$. Suppose that $f$ is in $L^{1}(G)$ and $\hat{f}(\gamma)=0$ whenever $\gamma$ is in $I$ but not in $B$. Let $E$ be a totally ordered subset of $B \cap I$. Then $\sum_{E}|\hat{f}(\gamma)|^{2} \leqq$ $4 K^{2}\|f\|_{1}^{2}$. The constant $K$ is the one appearing in the definition of Sidon set and does not depend on $I$ or $E$.

Proof. Let $g$ be a trigonometric polynomial with $\hat{g}=0$ off $I$. Put $h=f * g$. Then $\hat{h}=0$ off $B \cap I$ and in particular off $B$. By the definition of Sidon set,

$$
\sum_{I}|\hat{g}(\gamma) \hat{f}(\gamma)|=\sum_{B}|\hat{h}(\gamma)| \leqq K\|h\|_{\infty} \leqq K\|f\|_{1} \cdot\|g\|_{\infty} .
$$

Since the trigonometric polynomials with coefficients supported on $I$ are dense in $C_{I}(G)$, (1) holds for all $g$ in $C_{I}(G)$. Putting $w(\gamma)=\hat{f}(\gamma)$ we have that $\|w\|_{I} \leqq K\|f\|_{1}$. By Theorem $4, \sum_{E}|w(\gamma)|^{2} \leqq 4 K^{2}\|f\|_{1}^{2}$.

Observe that in the above $\hat{f}$ is arbitrary off $I$.

CoRollary. Let $E=\left\{m_{n}\right\}_{n=1}^{\infty}$ be any Hadamard set of positive integers (i.e., there is $a \lambda>1$ so that $m_{n+1} \geqq \lambda m_{n}$ for all n). Suppose 
that $f \in L^{1}(T)$ and $\hat{f}(m)=0$ for all $m \geqq 0$ which are not in $E$. Then $\sum_{E}|\hat{f}(m)|^{2} \leqq K\|f\|_{1}^{2}$ for some constant $K$ depending on $E$.

Proof. It is well known that every Hadamard set is a Sidon set. Theorem 10 applies with $I=S=\{m \mid m \geqq 0\}$ and $B=E$.

As a Sidon set need not be a Hadamard set Theorem 10 generalizes Theorem 1.

If $S$ is a half space, it is not necessary to know that $E$ is a Sidon set to obtain the conclusion of Theorem 10 .

Definition. A set $E \subset \Gamma$ is said to be of type $\Lambda(s), s>0$, if for some $r<s$, there is a constant $B_{r s}$ so that $\|f\|_{s} \leqq B_{r s}\|f\|_{r}$ for every trigonometric polynomial $f$ whose coefficients are 0 off $E$.

In [9, Th. 1.4], Rudin shows that if there is such a constant $B_{r s}$ for one $r<s$, then there are such constants $B_{r^{\prime} s}$ for all $r^{\prime}<s$.

The following argument was shown to us by F. Forelli. It resembles the one used by Rudin in proving Paley's theorem for half spaces [8, p. 222], and is the technique mentioned at the end of $\S 2$.

THEorem 11. Suppose that $S$ is a half-space, that is, that $\Gamma$ is totally ordered. Let $E \subset S$ be a $\Lambda$ (2) set. Then there is a constant $K$ so that $\sum_{E}|\hat{f}(\gamma)|^{2} \leqq K\|f\|_{1}^{2}$ for every $f$ with $\hat{f}=0$ on $S \sim E$.

Proof. First suppose that $f$ is a trigonometric polynomial. Let $g(x)=\sum_{S} \hat{f}(\gamma) \gamma(x)$ be the analytic projection of $f$. There is a constant $K_{1}$ so that $\|g\|_{1 / 2} \leqq K_{1}\|f\|_{1}[8$, Th. 8.7.6]. The coefficients of $g$ vanish off $E$ so that $\|g\|_{2} \leqq B_{(1 / 2) 2}\|g\|_{1 / 2} \leqq K_{2}\|f\|_{1}$, say. Then

$$
\sum_{E}|\hat{f}(\gamma)|^{2}=\|g\|_{2}^{2} \leqq\left(K_{2}\right)^{2}\|f\|_{1}^{2},
$$

the desired result with $K=\left(K_{2}\right)^{2}$.

We obtain the same conclusion for arbitrary $f$ by convoluting $f$ with a sequence of trigonometric polynomials which form an approximate identity.

Every Sidon set is of type $\Lambda(2)[8, \S 5 \cdot 7 \cdot 7]$. So when $S$ is a half space and $I=S$, Theorem 10 is a special case of Theorem 11 . When $S$ is smaller than a half space, however, the proof of 11 breaks down for the same reason as Rudin's proof of Paley's theorem: The analytic projection may not be a bounded operator from $L^{1}$ to $L^{r}$ for $r<1$.

One reason for considering theorems like these is that by an argument due to Banach [1, Th. a], they are equivalent to theorems about interpolating $l^{2}$ sequences by Fourier coefficients of continuous functions. We demonstrate this idea by applying it to Theorem 10 . 
THeOREM 12. Let $B$ be a Sidon set and $I$ a convex subset of $\Gamma$. Let $E$ be a totally ordered subset of $B \cap I$ and suppose that a sequence $v$ is defined on $E$ so that $\sum_{E}|v(\gamma)|^{2}<\infty$. Then there is a function $f$ in $C_{I}(G)$ with $\hat{f}(\gamma)=v(\gamma)$ for all $\gamma$ in $E$. Moreover $f$ can be chosen with $\|f\|_{\infty} \leqq 2 K^{\prime}\|v\|_{2}$ for any fixed $K^{\prime}$ larger than the constant $K$ associated with $B$.

Proof. Let $D$ be the closed subspace of elements $f$ of $C_{I}$ with $\hat{f} \equiv 0$ on $E$. Consider the bounded linear operator $L: C_{I} / D \rightarrow l^{2}(E)$ defined by $L([f])=\{\hat{f}(\gamma)\}_{\gamma \in E}$. We must show that $L$ is onto and that $\left\|L^{-1}\right\| \leqq 2 K$. The range of $L$ is dense in $l^{2}(E)$ and $L$ is one so that $L$ is onto if and only if $L^{*}$ is [8, p. 259, C 11].

Now $\left(C_{I} / D\right)^{*}=D^{\perp}$ the annihilator of $D$ in $\left(C_{I}\right)^{*}$. Also $\left(C_{I}\right)^{*}=$ $M(G) /\left(C_{I}\right)^{\llcorner}$where $M(G)$ is the space of bounded regular Borel measures on $G$ and $\left(C_{I}\right)^{\perp}$ is the set of such measures $\mu$ for which $\int f(x) d \mu(-x)=$ 0 for all $f$ in $C_{I}$. Since the trigonometric polynomials in $C_{I}$ are dense, $\left(C_{I}\right)^{\perp}=\{\mu$ in $M(G) \mid \hat{\mu}=0$ on $I\}$. Then $D^{\llcorner}=\left\{\mu+\left(C_{I}\right)^{\llcorner} \mid \hat{\mu}=0\right.$ off $\left.E\right\}$.

To any $l^{2}(E)$ sequence $w$ associate the $L^{2}(G)$ function $g(x)=$ $\sum_{\gamma \in E} w(\gamma) \gamma(x) . \quad L^{*}(w)$ is the coset $g+\left(C_{I}\right)^{\perp}$ in $D^{\perp}$.

Pick $\mu$ in $\left(C_{I}\right)^{\perp}$ and a finite subset $F$ of $E$. Let $P$ be a trigonometric polynomial with $\hat{P}=1$ on $F$. Then the function $h \equiv(g+\mu) * P$ is a trigonometric polynomial. On $I, \hat{\mu}(\gamma)=0$ so that $\hat{h}(\gamma)=\hat{g}(\gamma) \hat{P}(\gamma)$. In particular $\hat{h}(\gamma)=0$ on $I \sim B$. By Theorem 10 ,

$$
\sum_{F}|w(\gamma)|^{2}=\sum_{F}|\hat{h}(\gamma)|^{2} \leqq 4 K^{2}\|h\|_{1}^{2} .
$$

But $\left\|h_{1}\right\| \leqq\|g+\mu\| \cdot\|P\|_{1}$ and $\|P\|_{1}$ can be taken arbitrarily close to 1. Therefore $\sum_{F}|w(\gamma)|^{2} \leqq 4 K^{2}\|g+\mu\|^{2}$ for all finite subsets $F$ of $E$. Hence $\|g+\mu\| \geqq(1 / 2 K)\left(\sum_{E}|w(\gamma)|^{2}\right)^{1 / 2}$ for all $\mu$ in $\left(C_{I}\right)^{\llcorner}$. That is,

$$
\begin{aligned}
\left\|L^{*}(w)\right\| & =\inf \|g+\mu\| \quad\left(\mu \in\left(C_{I}\right)^{\perp}\right) \\
& \geqq(1 / 2 K)\|w\|_{2} .
\end{aligned}
$$

This means that $L^{*}$ is onto $[8$, p. $259, \mathrm{C} 11]$ and $\left\|\left(L^{*}\right)^{-1}\right\| \leqq 2 K$. Therefore $L$ is onto.

Moreover $\left\|L^{-1}\right\|=\left\|\left(L^{-1}\right)^{*}\right\|=\left\|\left(L^{*}\right)^{-1}\right\| \leqq 2 K$.

A similar interpolation theorem can be derived from Theorem 11 . For the circle group, for instance, it is well known that if $B$ is a Sidon set of integers and $v$ is a $l^{2}(B)$ sequence then there is a continuous $f$ with $\|f\|_{\infty} \leqq 2 K\|v\|_{2}$ and $\hat{f}(n)=v(n)$ on $B$ [10, Th. 5.1]. Also if $I=\left\{n \mid n_{1} \leqq n \leqq n_{2}\right\}$ and $v$ is 0 off $I \cap B$ then the trigonometric polynomial $g(\theta)=\sum_{I \cap B} v(n) \exp ($ in $\theta)$ has the right coefficients but, as $B$ is a Sidon set, $\|g\|_{\infty} \geqq(1 / K) \sum_{I \cap B}|v(n)|$, which may be much larger 
than $2 K\|v\|_{2}$. So the interpolating continuous function, in order to have small norm, may need some nonzero coefficients off $B$. Theorem 12 says that such an $f$ can still be taken as a trigonometric polynomial with coefficients supported by the smallest interval $I$ containing the support of $v$.

This paper is based on my $\mathrm{Ph}$. D. dissertation at the University of Wisconsin. Many of the ideas arose in conversations with various faculty members there. I would especially like to thank Prof. Frank Forelli for suggesting the problem and supervising my research.

\section{REFERENCES}

1. S. Banach, Uber eine Eigenschaft der lacunären trigonometrischen Reihen, Studia Math. 2 (1930), 207-220.

2. H. Bohr, Uber, die Bedeutung der Potenzreihen unendlich vieler Variabeln in der Theorie der Dirichletchen Reihen $\Sigma a_{n} / n^{s}$, Nach. Akad. Wiss. Göttingen Math. -Phys. Kl. 2 (1913), 441-488.

3. N. Fine, On the Walsh functions, Trans, Amer. Math. Soc., 65 (1949), 372-414.

4. Henry Helson, Conjugate series and a theorem of Paley, Pacific J. Math. 8 (1958), 437-446.

5. J. E. Littlewood, On the mean values of power series (II), J. London Math. Soc. (1930), 179-182.

6. R.E.A.C. Paley, A note on power series, J. London Math. Soc. 7 (1932), 122-130.

7. Daniel Rider, $A$ relation between a theorem of Bohr and Sidon sets, Bull. Amer. Math. Soc. 72 (1966), 558-561.

8. Walter Rudin, Fourier analysis, on groups, Interscience, New York, 1962.

9. — Remarks on a theorem of Paley, J. London Math. Soc. 32 (1957), 307-311.

10. - Trigonometric series with gaps, J. Math. Mech. 9 (1960), 203-227.

11. S. B. Steckin, An extremal problem for polynomials, Izv. Akad. Nauk. SSSR Ser. Math. 20 (1956), 765-774; Amer. Math. Soc. Translations, Series. 2, Vol. 14, 173-180. 12. E. M. Stein, On limits of sequences of operators, Ann. of Math. (2) 74 (1961), $140-170$.

13. A. Zygmund, On the boundary values of functions of several complex variables, I, Fund. Math. 36 (1949), 207-235.

14. — Trigonometric series, 2nd ed., Vol. I, Cambridge University Press, Cambridge, 1959.

Received January 26, 1968.

UNIVERSITY OF BRITISH COLUMBIA 



\title{
PACIFIC JOURNAL OF MATHEMATICS
}

\author{
EDITORS
}

H. ROYDEN

Stanford University

Stanford, California

R. R. PhelPS

University of Washington

Seattle, Washington 98105
J. DUGUNDJI

Department of Mathematics

University of Southern California

Los Angeles, California 90007

RICHARD ARENS

University of California

Los Angeles, California 90024

\section{ASSOCIATE EDITORS}
E. F. BECKENBACH
B. H. NeumanN
F. WOLF
K. YoSHIDA

\section{SUPPORTING INSTITUTIONS}

\author{
UNIVERSITY OF BRITISH COLUMBIA \\ CALIFORNIA INSTITUTE OF TECHNOLOGY \\ UNIVERSITY OF CALIFORNIA \\ MONTANA STATE UNIVERSITY \\ UNIVERSITY OF NEVADA \\ NEW MEXICO STATE UNIVERSITY \\ OREGON STATE UNIVERSITY \\ UNIVERSITY OF OREGON \\ OSAKA UNIVERSITY \\ UNIVERSITY OF SOUTHERN CALIFORNIA
}

\author{
STANFORD UNIVERSITY \\ UNIVERSITY OF TOKYO \\ UNIVERSITY OF UTAH \\ WASHINGTON STATE UNIVERSITY \\ UNIVERSITY OF WASHINGTON \\ $\stackrel{*}{*} \stackrel{*}{*}{ }^{*}{ }^{*}$ \\ CHEVRON RESEARCH CORPORATION \\ TRW SYSTEMS \\ NAVAL WEAPONS CENTER
}

The Supporting Institutions listed above contribute to the cost of publication of this Journal, but they are not owners or publishers and have no responsibility for its content or policies.

Mathematical papers intended for publication in the Pacific Journal of Mathematics should be in typed form or offset-reproduced, double spaced with large margins. Underline Greek letters in red, German in green, and script in blue. The first paragraph or two must be capable of being used separately as a synopsis of the entire paper. It should not contain references to the bibliography. Manuscripts, in duplicate if possible, may be sent to any one of the four editors. Please classify according to the scheme of Math. Rev. 36, 1539-1546. All other communications to the editors should be addressed to the managing editor, Richard Arens, University of California, Los Angeles, California, 90024.

50 reprints are provided free for each article; additional copies may be obtained at cost in multiples of 50 .

The Pacific Journal of Mathematics is published monthly. Effective with Volume 16 the price per volume (3 numbers) is $\$ 8.00$; single issues, $\$ 3.00$. Special price for current issues to individual faculty members of supporting institutions and to individual members of the American Mathematical Society: $\$ 4.00$ per volume; single issues $\$ 1.50$. Back numbers are available.

Subscriptions, orders for back numbers, and changes of address should be sent to Pacific Journal of Mathematics, 103 Highland Boulevard, Berkeley, California, 94708.

PUBLISHED BY PACIFIC JOURNAL OF MATHEMATICS, A NON-PROFIT CORPORATION

Printed at Kokusai Bunken Insatsusha (International Academic Printing Co., Ltd.), 7-17, Fujimi 2-chome, Chiyoda-ku, Tokyo, Japan. 


\section{Pacific Journal of Mathematics \\ Vol. 30, No. $2 \quad$ October, 1969}

Gregory Frank Bachelis, Homomorphisms of annihilator Banach algebras.

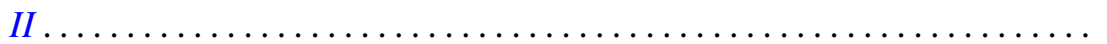

Leon Bernstein and Helmut Hasse, An explicit formula for the units of an algebraic number field of degree $n \geq 2 \ldots \ldots \ldots \ldots \ldots \ldots \ldots . \ldots 29$

David W. Boyd, Best constants in a class of integral inequalities ........ 367

Paul F. Conrad and John Dauns, An embedding theorem for lattice-ordered

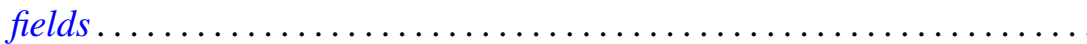

H. P. Dikshit, Summability of Fourier series by triangular matrix

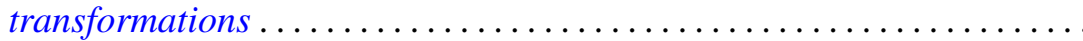

Dragomir Z. Djokovic, Linear transformations of tensor products preserving a fixed rank............................. 411

John J. F. Fournier, Extensions of a Fourier multiplier theorem of Paley . . . 415 Robert Paul Kopp, A subcollection of algebras in a collection of Banach

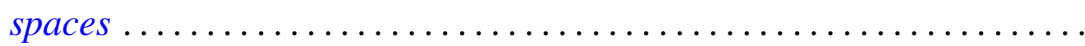

Lawrence Louis Larmore, Twisted cohomology and enumeration of vector bundles ...................................... 437

William Grenfell Leavitt and Yu-Lee Lee, A radical coinciding with the lower radical in associative and alternative rings .................

Samuel Merrill and Nand Lal, Characterization of certain invariant subspaces of $H^{p}$ and $L^{p}$ spaces derived from logmodular

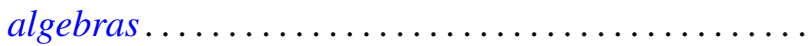

Sam Bernard Nadler, Jr., Multi-valued contraction mappings ....

T. V. Panchapagesan, Semi-groups of scalar type operators in Banach spaces ....................................

J. W. Spellmann, Concerning the infinite differentiability of semigroup motions

H. M. (Hari Mohan) Srivastava, A note on certain dual series equations involving Laguerre polynomials.

Ernest Lester Stitzinger, A nonimbedding theorem of associative algebras................................

J. Jerry Uhl, Jr., Martingales of vector valued set functions ...

John Mays Worrell Jr., On continuous mappings of metacompact $\check{C} e c h$

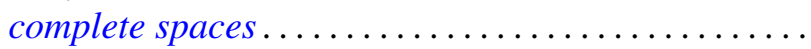

